\title{
Modulation of current through a nanopore induced by a charged globule: implications for DNA-docking
}

\author{
Mauro Chinappi ${ }^{1}$, Carlo Massimo Casciola ${ }^{2,3}$, Fabio Cecconi ${ }^{4}$, Umberto Marini Bettolo Marconi ${ }^{5}$ \\ and Simone Melchionna ${ }^{6}$ \\ 1 Center for Life Nano Science@Sapienza, Istituto Italiano di Tecnologia, Via Regina Elena 291, 00161, Roma, Italia \\ 2 Dipartimento di Ingegneria Meccanica e Aerospaziale, Sapienza Università di Roma, Via Eudossiana 18, 00184, \\ Roma, Italia \\ 3 Center for Life Nano Science@Sapienza, Istituto Italiano di Tecnologia, Via Regina Elena 291, 00161, Roma, Italia \\ 4 CNR-Istituto dei Sistemi Complessi, Via Dei Taurini 19, 00185 Roma, Italy \\ 5 Scuola di Scienze e Tecnologie, Università di Camerino, Via Madonna delle Carceri, 62032 Camerino, INFN, sez. \\ di Perugia, Italy \\ ${ }^{6}$ CNR-IPCF, Consiglio Nazionale delle Ricerche, Dipartimento di Fisica, Università Sapienza, Piazzale A. Moro 2, \\ 00185 Roma, Italy
}

PACS 66.90. $+r-$ Other topics in nonelectronic transport properties of condensed matter

PACS 66.10.Ed - Ionic conduction

PACS $61.25 . \mathrm{H}--$ Macromolecular and polymers solutions; polymer melts

\begin{abstract}
The passage of DNA through a nanopore can be effectively decomposed into two distinct phases, docking and actual translocation. In experiments each phase is characterized by a distinct current signature which allows the discrimination of the two events. However, at low voltages a clear distinction of the two phases is lost. By using numerical simulations we clarify how the current signature associated to the docking events depends on the applied voltage. The simulations show that at small voltage the DNA globule enhances the pore conductance due to an enrichment of charge carriers. At high voltage, the globule drains substantial charge carriers from the pore region, thereby reducing the overall conductance. The results provide a new interpretation to the experimental data on conductance and show how docking interferes with the translocation signal, of potential interest for sequencing applications.
\end{abstract}

Introduction. - In the last decade, nanopore-based biosensing has become a burgeoning research field thanks to the impressive burst in the capability to fabricate devices based on solid state [1,2] and biological pores [3 [5]. The working principle of the device is, in essence, simple. The nanopore connects two chambers containing an electrolyte solution. Under an electric voltage, ions migrate from one chamber to the other, with a conductance that depends on the details of the pore and the electrolytic solution. Since a translocating macromolecule alters the ionic flux, its passage can be detected and possibly the local nature of its monomers can be read off. Translocation of DNA is the most studied process [6] being a promising technology for low cost/high throughput DNA analysis, its realization is still confronted with challenges 7-10. In parallel, a separate set of studies have focused on protein and polypeptide translocation, with the goal of structural characterization $11-15$.

Over the pore extension, the DNA segment has a virtually rod-like conformation and during the threading process the ionic current increases for low salt $(<0.4 \mathrm{M} \mathrm{KCl})$ and decreases for high salt concentration [16, 17] as compared to the open pore (so called free-pore in the following) case. The accepted explanation for the current reduction is that at high salinity the ionic motion is sterically hampered by the presence of DNA that reduces the effective pore section [18]. At low salinity instead, the counterions cloud is more diffused and the charge carriers more mobile, an effect that prevails over the steric hinderance, increasing the conductance as compared to the free-pore condition 16. 17 .

Recently, Kowalczyk and Dekker [8] and Vlassarev and Golovchenko [19 reported of a new type of hybrid event with two distinct signals, a current decrease followed by a 


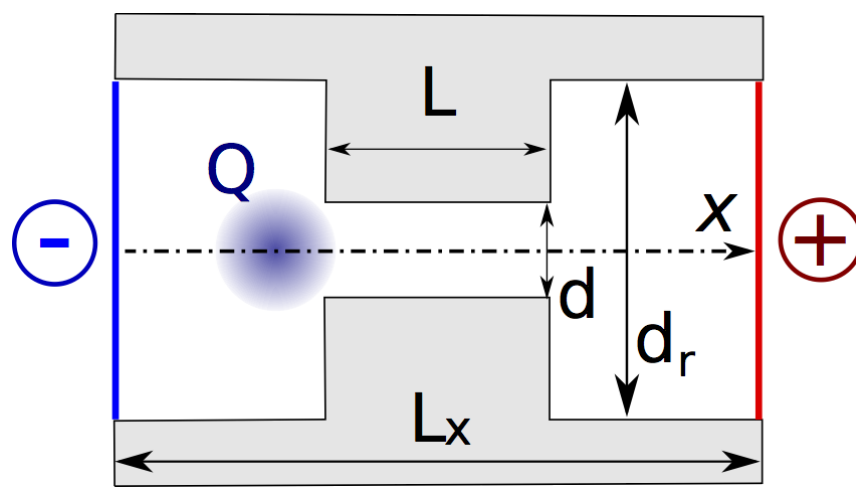

Figure 1: A) Sketch of the system. A cylindrical pore of length $L$ and diameter $d$ connects two reservoirs of diameter $d_{r}$, with total length $L_{x}=L+2 L_{r}$, and aligned along the $x$ axis. Bulk concentrations and the electric potential are imposed at the left $(-)$ and right $(+)$ boundaries. An immobile globule of total negative charge $Q$ is located near the pore entrance. Note that the sketch does not reflect the actual aspect ratio of the system, see Fig. 2

current increase. Interestingly, the amount of current reduction is comparable to that of current enhancement and the transition between the two regimes takes place on a timescale shorter than the translocation event. The experiments were performed in low salt conditions and, hence, the increase in current was associated to the threading of DNA, while the decrease of current was interpreted as due to the presence of the DNA at the pore entrance. In fact, since the electric field is highly focused in the pore and access regions, the modulation of ionic current takes place only when DNA is in close proximity to the pore, in the so-called docked configuration. In addition, current reduction was attributed to the frictional forces exerted by the docked DNA on the solution 8]. However, the physical mechanism responsible for decreasing the ionic current remains elusive. In fact, while frictional forces can reduce electrokinetic transport, the transition between docked and threading DNA should be gradual. If friction has a leading role, a decrease of conductance should persist during DNA threading, since large portions of the DNA coil sit on both the cis and trans chambers of the device.

Assessing the contribution to conductance stemming from a docked DNA is relevant to correctly discriminate the ionic signal arising from the translocating nucleotides. Due to the comparable intensity of ionic currents associated to docked and translocating conformations, subtracting the systematic contribution originating from partially translocated DNA globules is particularly useful in the low voltage regime, when translocation is slow and therefore the base pair content is amenable to detection. The present study aims at understanding the origin of current modulation by employing computer simulations in a model representation of the system. We consider an idealized version of the device with DNA modelled as a charged

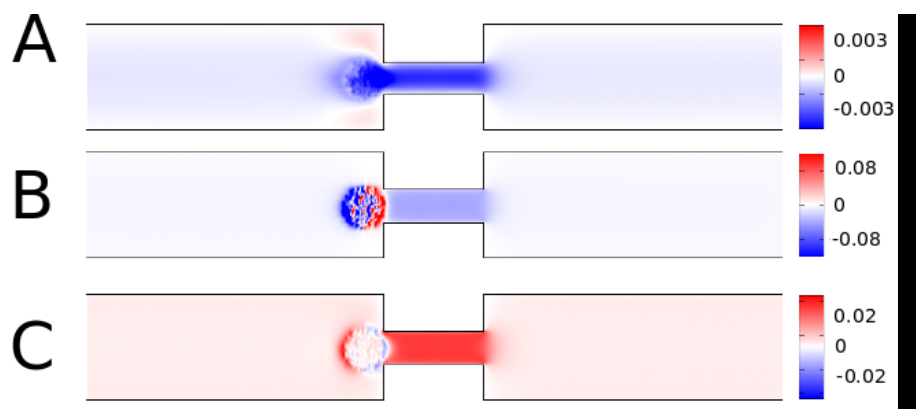

Figure 2: The current fields $J^{n}, J^{+}$and $J^{-}$, for the neutral, positive and negative species, on the $\mathrm{y}-\mathrm{z}$ plane. A: $J_{x}^{n}$, driven by electro-osmosis, on average directed $(\leftarrow+)$, B: $J_{x}^{+}$, on average directed $(\leftarrow+)$ and $\mathrm{C}: J_{x}^{-}$, on average directed $(\rightarrow$ $+)$. Currents are reported in units of $\rho_{b u l k}^{\alpha} v_{T}$

globule docked near the pore entrance.

Our results reproduce qualitatively the modulation of current at varying voltages as observed in experiments. In addition, they suggest an alternative cause for the observed modulation that is based entirely on electrokinetic effects. In particular, i) a substantial depletion of ions takes place within the pore due to the asymmetric charge drainage exerted by the globule, ii) the current decrease can be explained in terms of charge rearrangements, without the need for considering excluded volume or hydrodynamics interactions between DNA and the pore, iii) at low voltage the current increases as compared to the free-pore case, a regime that has not been detected by experiments. We further suggest that at low voltages the current increase observed in experiments should be assigned to hybrid docked/translocating configurations.

System set-up and simulation method. - We consider a cylindrical nanopore of length $L$ and diameter $d=0.3 L$ connecting two reservoirs of length $L_{r}=3 L$ and diameter $d=L$, (Fig. 1). The origin of the coordinate system is located at the center of the pore and the $\mathrm{x}$-axis coincides with the pore axis. A potential difference $\Delta V$ is applied between a positive and a negative electrode, placed at $x= \pm 3.5 L$, respectively. The fluid is composed of three species: positive charge carriers, negative charge carriers and a neutral species 20,21]. The density fields are indicated as $\rho^{+}, \rho^{-}$and $\rho^{n}$ respectively. The electrolytic solution is assumed to be neutral and homogeneous far away from the pore, with $\rho_{\text {bulk }}^{+}=\rho_{\text {bulk }}^{-}$at the two open boundaries $(x= \pm 3.5 L)$. The Bjerrum and Debye lengths are set to $l_{B}=0.035 L$ and $\lambda_{D}=0.05 L$, respectively. These values are chosen in order to map the conditions employed in the experiments 817

The role of the wall charge in the translocation experiment is two-fold: to enhance the electro-osmotic current

\footnotetext{
${ }^{1}$ In Ref. 8. $L \simeq 20 \mathrm{~nm}$ and $d \simeq 6 \mathrm{~nm}$ while, $l_{B}=0.7 \mathrm{~nm}$ and $l_{D}=10 \mathrm{~nm}$, corresponding to a $0.15 \mathrm{M}$ solution of a monovalent salt in water.
} 
Current through a nanopore induced by a charged globule

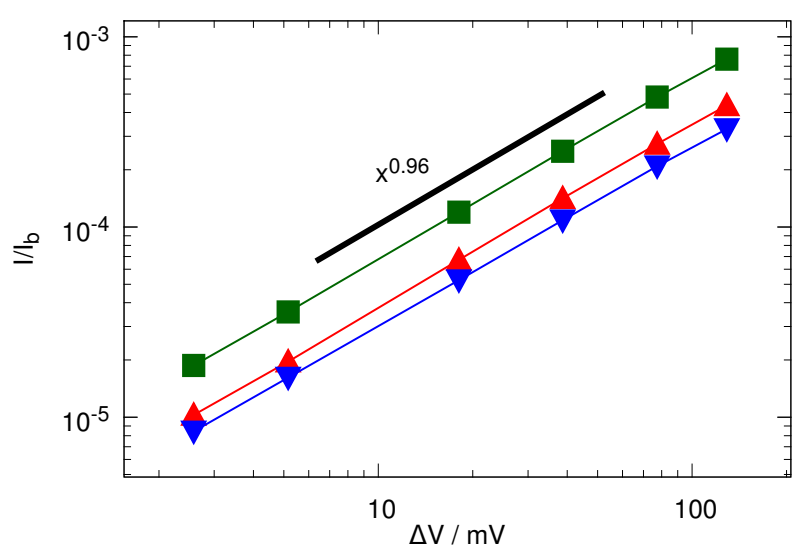

Figure 3: Positive $\left(\left|I^{+}\right|\right.$, triangles $)$and negative $\left(\left|I^{-}\right|\right.$, inverted triangles) current intensities as functions of the applied voltage. Squares refer to the electrical current intensity $\left(I_{c}=\left|I^{+}-I^{-}\right|\right)$. A non linear dependence of the electrical current intensity is apparent $\left(I_{c} \sim \Delta V^{\alpha}\right.$ with $\left.\alpha=0.93 \pm 0.01\right)$. The current is represented in units of $I_{b}=\pi\left(d_{r} / 2\right)^{2} \rho_{b u l k}^{+} / v_{T}$.

by altering the amount of free charge carriers via the Donnan effect and to introduce additional interactions with the macromolecule 15,22 . In the present work we neglect the presence of surface wall charge in order to keep the number of simulation parameters to a bare minimum and to isolate the effect of the docking globule on the ionic current. Such approximation allows capturing the leading effects in the modulation of the ionic conduction, while the presence of additional free charge carriers can be accounted for by modifying the bulk molarity. Secondly, as we are interested in docking conditions, the surface charge is expected to play a minor role on the DNA globule and charge redistribution outside the pore region.

The presence of DNA docked at the pore entrance is mimicked by a spherical distribution of negative charges is positioned in a fixed location near the pore entrance, the total globule charge being $Q$ (Fig. 1).

The motion of the electrolytic solution is described by the density, velocity and electric fields of each species, having number densities $\rho^{\alpha}=\rho^{n}, \rho^{+}, \rho^{-}$, equal unit mass, valence $z^{\alpha}=0,1,1$, and equal mobilities. We adopt the continuum approach, implicitly assuming that the motion of individual molecules is not relevant for the problem at hand. Several studies indicate that for simple fluids this is a good description down to the nanoscale 23, 24]. The electrokinetic flow is simulated by means of the Lattice Boltzmann Method, which reproduces the advectiondiffusion dynamics for three species, a neutral one representing the solvent, and two charged species for the electrolytes, in the presence of electrostatic interactions 20, 21, 25. Simulations were performed with the parallel multiphysics software MUPHY 26 .

Atomistic simulations and recent experiments indicate that the slip length $L_{s}$ for water on smooth surfaces hardly exceeds $1 \mathrm{~nm}$ [27, 28], therefore no-slip boundary condi-

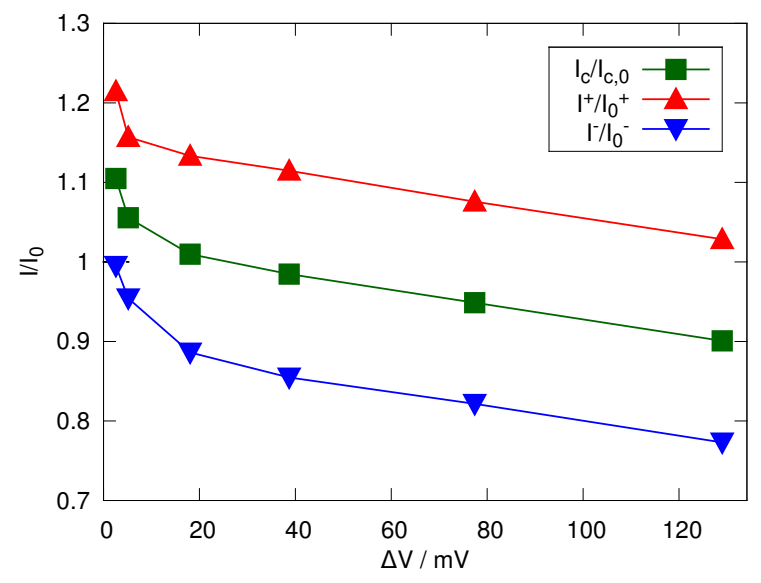

Figure 4: Current in the presence of the globule at the pore entrance normalized by the value in absence of the globule, as a function of the applied voltage. Blue inverted triangle and red triangles refer to negative and positive carriers respectively, while green squares refer to the total electric current.

tions are applied for each fluid species at the solid wall 27, 28. The materials usually employed for fabricating the nanopore are hydrophilic, thus allowing to safely disregard slip effects at the scale of interest. For electrostatics, the Poisson equation, $\nabla^{2} \phi=-\frac{1}{\epsilon}\left(e \rho^{+}-e \rho^{-}+\theta\right)$, where $\theta$ is the globule charge distribution, is solved with appropriate boundary conditions. The tangential component of the electric field is continuous at the liquid-solid interface. Given the large disparity in the dielectric constants for water $(\epsilon \simeq 80)$ and the wall $(\epsilon \simeq 3.9$ for silicon oxide and $\simeq 7.9$ for silicon nitride), we impose Neumann boundary conditions, $\nabla \Phi \cdot \hat{\mathbf{n}}=0$. where $\hat{\mathbf{n}}$ is the unit vector orthogonal to the wall. Molecular Dynamics simulations have shown that the dielectric constant of water confined in a cavity of diameter $5 \mathrm{~nm}$ is 71 , close to the value in the bulk 29]. Hence, given the size of our system $(d=6 \mathrm{~nm})$, a minor alteration of the dielectric constant is expected and we employed $\epsilon=80$ in the whole system.

The docked globule is represented as a system of regularized point-wise charges, each carrying a unit charge $0.1 e$, and distributed uniformly within a sphere of diameter $d$, i.e. the same diameter of the pore. The interaction strength is given by the Bjerrum length, $l_{B}=e^{2} / 4 \pi \epsilon k_{B} T$, which is taken to be $0.7 \mathrm{~nm}$, corresponding to liquid water conditions at ambient temperature. In order to resolve the double layer structure and avoid compressibility artifacts arising from the numerical method, we set the spacing of the computational grid to $0.02 \mathrm{~nm}$. Fully developed flow is assumed at the inlet and at the outlet sections, each having a prescribed electrical potential $\left(\phi_{i n}=0\right.$ and $\left.\phi_{\text {out }}=\Delta V\right)$. Dirichlet boundary conditions enforce the bulk densities of each species at the inlet and outlet, in particular, $\rho_{\text {in }}^{+}=\rho_{\text {out }}^{+}=\rho_{\text {in }}^{-}=\rho_{\text {out }}^{-}$.

For the Lattice Boltzmann method, the electrolytic solution is represented by three distribution functions, 
$f^{\alpha}(x, v, t)$, representing the probability of having a particle of species $\alpha$ at position $x$, with velocity $v$ and at time $t$. The species evolve according to the equation

$\partial_{t} f^{\alpha}+\nabla \cdot v f^{\alpha}=\omega\left(f_{e q}^{\alpha}-f^{\alpha}\right)+\frac{e z^{\alpha}}{m} \nabla \phi \cdot \frac{\partial}{\partial v} f^{\alpha} \equiv C^{\alpha}[\{f\}]$

where $f_{e q}^{\alpha}=\left[\frac{1}{2 \pi v_{T}^{2}}\right]^{3 / 2} \rho^{\alpha} e^{-\frac{(v-u)^{2}}{2 v_{T}^{2}}}$ is the MaxwellBoltzmann local equilibrium, $v_{T}$ the thermal velocity, $u=\frac{\sum_{\alpha} \rho^{\alpha} u^{\alpha}}{\sum_{\beta} \rho^{\beta}}$ is the barycentric fluid velocity and $u^{\alpha}$ the species velocity. The quantity $\omega$ controls the rate of relaxation towards local equilibrium and is related to the kinematic viscosity $\nu$ as $\nu=v_{T}^{2}(1 / \omega-1 / 2)$. The distributions $f^{\alpha}$ are discretized in space over a Cartesian mesh and expanded in velocity space over a Hermite basis set. By Hermite projection, the distributions are replaced by $f^{\alpha}(x, v, t) \rightarrow f_{p}^{\alpha}$ and analogously $C^{\alpha}(x, v, t) \rightarrow C_{p}^{\alpha}$. The dynamics is generated according to the updating scheme

$$
f_{p}^{\alpha}\left(x+c_{p}, t+1\right)-f_{p}^{\alpha}(x, t)=C_{p}^{\alpha}(x, t)
$$

where $c_{p}$ represents a set of discrete speed that connects a mesh point to its neighbors. The species density is computed as $\rho^{\alpha}=\sum_{p} f_{p}^{\alpha}$ and the species current as $J^{\alpha} \equiv \rho^{\alpha} u^{\alpha}=\sum_{p} c_{p} f_{p}^{\alpha}$. Example of the obtained current fields are shown in Fig. 2

Results. - The current intensity of the species $\alpha$ is defined as $I^{\alpha}=\int_{A(x)} \rho^{\alpha} u_{x}^{\alpha} d A$ where $A(x)$ is the local section perpendicular to the pore axis, $\rho^{\alpha}$ is the number density of a given species and $u_{x}^{\alpha}$ the $\mathrm{x}$-component of the velocity field. The total ionic current is $I_{c}=I^{+}-I^{-}$. As a preliminary check, we verified that in stationary flow conditions the system is globally neutral, and that in the absence of the globule, the number of positive and negative carriers is exactly the same. Given the geometrical symmetry and the identical valence and mobility of the charged species the positive charge flow (electrodes $(+) \rightarrow(-)$ ) exactly balances the flow of negative carriers (electrodes $(-) \rightarrow(+)$ ), so that no electro-osmotic flow, i.e. the net motion of the neutral specie, is present. Moreover, the system response is found to be purely Ohmic and the ionic currents are individually proportional to the applied voltage $V$ (data not shown). In the following, we refer to quantities in the absence of the globule (free-pore case) with the subscript 0 .

The presence of the globule results in an abundance of positive carriers necessary to maintain the condition of global electroneutrality. The unbalance between positive and negative carriers reflects in a difference between $\left|I^{+}\right|$ and $\left|I^{-}\right|$, see Fig 3 .

To appreciate the role of the applied voltage, it is instructive to compare the data in the presence and in the absence of the globule. Fig. 4 reports the ratio between the current intensities for total globule charges $Q=100 e$ and $Q=0$. In the explored voltage range the presence of the globule at the pore entrance leads to an increase of positive carrier current, $I^{+} / I_{0}^{+}>1$ (red triangles in Fig. (4) and to a depletion of the negative carrier one, $I^{-} / I_{0}^{-}<1$ (blue inverted triangles in Fig. 4). This modulation is caused by the already mentioned abundance of positive carriers.

A second observation is that both $I^{+} / I_{0}^{+}$and $I^{-} / I_{0}^{-}$ decrease as the applied voltage $\Delta V$ increases, consistently with the depletion of carriers in the pore region as the voltage increases, see Fig. 5A. Interestingly at low voltages $I_{c} / I_{c, 0}>0$, i.e. the presence of the charged globule results in an increase of the current, while at high voltage $I_{c} / I_{c, 0}<0$. The conductance modulation as a function of $\Delta V$ is interpreted by considering the simple case of a globule placed in the middle of a cylindrical tube of length $L_{x}=L+2 L_{r}$ and diameter $d_{r}=L$, i.e. a single cylinder with the same diameter of the reservoir with no interposed nanopore, spanning the whole length of the system. At zero voltage, the charge distribution is symmetric around the globule and, outside the Debye layer (i.e. at distance $>3 \lambda_{D}$ from the globule), both $\rho^{+}$and $\rho^{-}$recover the bulk value imposed at the two open boundaries. Under a finite voltage, the counterion cloud surrounding the globule is asymmetrically distorted towards the negative electrode. The counterion density is therefore larger towards the negative electrode (charge surplus) than towards the positive electrode (charge depletion), see Fig. 5B. Clearly, outside the Debye layer the solution recovers local electroneutrality $\left(\rho^{+}=\rho^{-}\right)$, with an antisymmetric arrangement of charges with respect to the globule position induced by the applied voltage.

The presence of the pore breaks the fore-aft symmetry and results in a substantial carrier depletion inside the pore (i.e. towards the positive electrode with respect to the globule). Such depletion is one order of magnitude more intense than the corresponding increase towards the negative electrode, see Fig. 5A. The pore induces an imperfect electrostatic screening as compared on the opposite side of the globule. The effect increases with $\Delta V$ and hence, at high voltages, the carrier density inside the pore is strongly depleted by the charged globule.

In essence, the docked globule produces two concomitant effects: i) the global increase in the available charge carriers and ii) the local depletion of carriers inside the pore. At low $\Delta V$, the depleted pore plays a marginal role on the overall conduction and the leading effect is the increase of positive carriers, hence $I_{c}>I_{c, 0}$. At high $\Delta V$, the depleted pore dominates on the increase of the available counterions, hence $I_{c}<I_{c, 0}$.

It is illustrative to relate the results of the present simulations with experimental findings of Kowalczyk and Dekker [8]. We remind that these authors reported the intensity and the average duration of the current modulations, as shown in Fig. 6C. The current decrease was associated to docking while the current increases to translocation events. Let us now focus on the current decrease and the differential conductance, defined as $\Delta G \equiv$ $\left(I_{c}-I_{c, 0}\right) / \Delta V$. The experimental docking conductance 

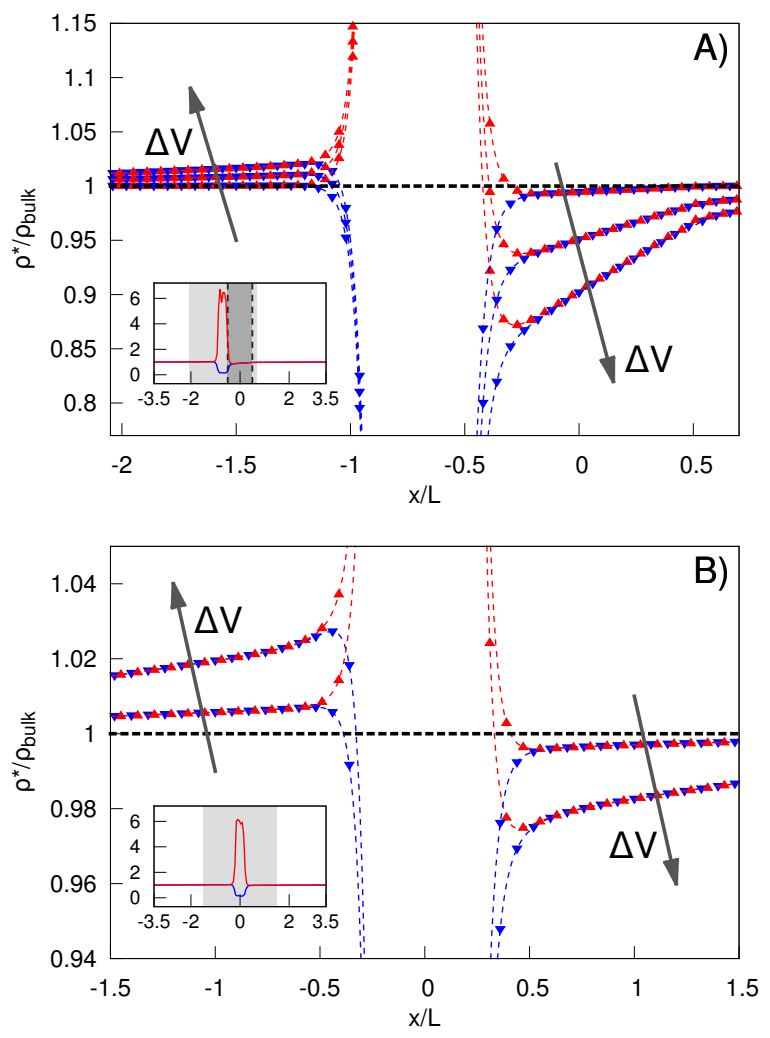

Figure 5: A: density profiles of positive (red triangles) and negative (blue inverted triangles) species normalized by the bulk value, for applied voltage $\Delta V=5.2,38.8,77.5 \mathrm{mV}$ (the arrows indicate the trend at increasing voltage). Inset: zoomout of the profiles, with the light shaded area showing the data reported in the main graph and the dark shaded area the pore region. B: same as in the upper panel but in absence of the pore $(\Delta V=18.1,77.5 \mathrm{mV})$.

decreases monotonically in the range $200 m V<\Delta V<$ $600 \mathrm{mV}$ (red squares in Fig. [6C) in qualitative agreement with our numerical result in the voltage range where $I_{c} / I_{c, 0}<1$. At small voltages $(\Delta V<200 m V)$, the experiments do not detect any current decrease, whereas the simulations show that $I_{c} / I_{c, 0}>1$. Moreover, experiments show an optimal enhancement at $V \simeq 125 \mathrm{mV}$ (black circles in Fig. 66). The apparent discrepancy between experiments and simulations at low voltages can be reconciled according to the following argument. For $200 m V<$ $\Delta V<600 m V$ the experiments detect hybrid events, with a clear-cut separation between reduction/docking (of duration $t_{d}$ ) and enhancement/translocation (of duration $t_{t}$ ) events. Two distinct signals, called $I_{d}$ and $I_{t}$ respectively, are measured as averages over the time intervals $t_{d}$ and $t_{t}$ (Fig. 6B). At low voltage, the simulations indicate that the ionic current is enhanced as compared to the free-pore case. Since both the docking and the translocation events feature an increase in conductance (Fig. 64A) the docking events are hardly distinguishable from the translocation ones. In particular, as voltage diminishes, the docking events become slower than the translocating ones, as reported in 8 .

To estimate the conductance for low $\Delta V$, we average the experimental data of 8 over docking and translocating events, as

$$
\Delta G=\frac{\Delta G_{d} t_{d}+\Delta G_{t} t_{t}}{t_{d}+t_{t}} .
$$

where $\Delta G_{d}, \Delta G_{t}, t_{d}$ and $t_{t}$ depend individually on the applied voltage. We evaluate the conductance in the range $50 \mathrm{mV}<\Delta V<125 \mathrm{mV}$ by using for $t_{d}$ and $t_{t}$ the expressions of ref. [8], and fit the experimental data in the interval $200 m V<\Delta V<600 m V$ to extrapolate the differential conductances $\Delta G_{d}$ and $\Delta G_{t}$ at low voltage. In passing, we note that the differential conductance due to docking events is zero for $\Delta V \simeq 130 \mathrm{mV}$. This value is comparable to the numerical one $(\simeq 30 \mathrm{mV})$, notwithstanding the approximations involved, in particular regarding the large disparity of the globule size between simulation and experiments and the absence of pore wall charge. The final result of the averaging of eq. (1) is reported in Fig. 6C (blue solid line), which exhibits a good agreement with the experimental result, thereby lending good confidence in the proposed interpretation.

In conclusion, the present work underscores the importance of electrokinetic effects on DNA translocation experiments. In particular, it indicates that DNA docked near the pore entrance can either enhance or reduce the pore conductance at different applied voltages as compared to the free-pore case. Such non trivial phenomenon depends on the imperfect electrostatic screening that depletes the pore region from charge carriers. On the other hand, as the macromolecule begins threading, the conductance is dominated by the DNA thread in the pore region that increases and compensates for the charge drainage exerted by the DNA coil at the pore entrance. In the future, it will be interesting to investigate the role played by the charged wall and how the interplay between coil induced drainage and thread induced enrichment affects ionic conductance during DNA translocation.

$$
* * *
$$

Computing time at the CINECA supercomputing center (ISCRA grants FLEXPROT and NAPS) is kindly acknowledged.

References

[1] Russo C. J. and Golovchenko J., Proceedings of the National Academy of Sciences, 109 (2012) 5953.

[2] Storm A., Chen J., Ling X., Zandbergen H. and Dekker C., Nature materials, 2 (2003) 537.

[3] Hall A. R., Scott A., Rotem D., Mehta K. K., BaYLEY H. and DekKer C., Nature nanotechnology, 5 (2010) 874 .

[4] Mohammad M., Iyer R., Howard K., McPike M., Borer P. and Movileanu L., Journal of the American Chemical Society, 134 (2012) 9521. 

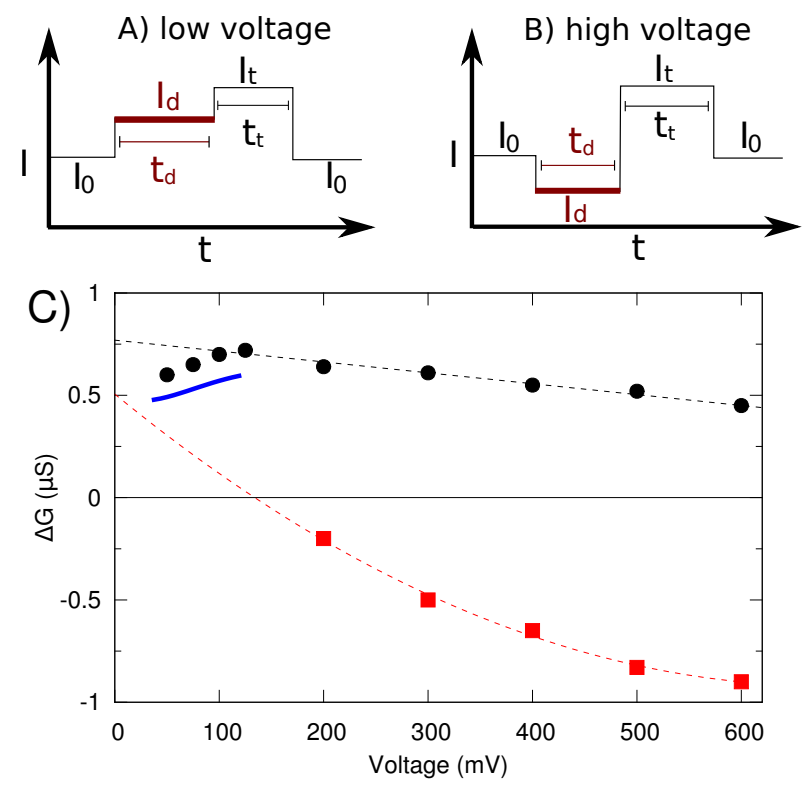

Figure 6: Upper panels: low (a) and high (b) voltage scenarios. At low voltage both docking and translocation events increase the current. Lower panel: experimental differential conductance [8], associated to docking (red squares) and translocation (black circles). The dashed curves are fits of the experimental data for $200 m V<\Delta V<600 m V$. The blue line is the new estimate, eq. (3), for $50 m V<\Delta V<125 m V$.

[5] Laszlo A. H., Derrington I. M., Brinkerhoff H., Langford K. W., Nova I. C., Samson J. M., Bartlett J. J., Pavlenok M. and Gundlach J. H., Proceedings of the National Academy of Sciences, 110 (2013) 18904.

[6] Kasianowicz J. J., Brandin E., Branton D. and Deamer D. W., Proceedings of the National Academy of Sciences, 93 (1996) 13770.

[7] Pennisi E., Science, 343 (2014) 829.

[8] Kowalczyk S. W. and Dekker C., Nano letters, 12 (2012) 4159.

[9] Fyta M. G., Melchionna S., Kaxiras E. and Succi S., Multiscale Modeling \& Simulation, 5 (2006) 1156.

[10] Schneider G. and DekKer C., Nature biotechnology, 30 (2012) 326.

[11] Rodriguez-Larrea D. and Bayley H., Nature nanotechnology, (2013).

[12] Bacci M., Chinappi M., Casciola C. and Cecconi F., The Journal of Physical Chemistry B, 116 (2012) 4255.

[13] Bacci M., Chinappi M., Casciola C. M. and Cecconi F., Physical Review E, 88 (2013) 022712.

[14] Madampage C., Tavassoly O., Christensen C., Kumari M. and Lee J., Prion, 6 (2012) 110.

[15] Cressiot B., Oukhaled A., Patriarche G., Pastoriza-Gallego M., Betton J., Auvray L., Muthukumar M., Bacri L. and Pelta J., ACS nano, 6 (2012) 6236.

[16] Chang H., Kosari F., Andreadakis G., Alam M., Vasmatzis G. and Bashir R., Nano Letters, 4 (2004) 1551.

[17] Fan R., Karnik R., Yue M., Li D., Majumdar A. and
YANG P., Nano Letters, 5 (2005) 1633.

[18] Smeets R. M., Keyser U. F., Krapf D., Wu M.-Y., Dekker N. H. and Dekker C., Nano Letters, 6 (2006) 89.

[19] Vlassarev D. M. and Golovchenko J. A., Biophysical Journal, 103 (2012) 352.

[20] Marini Bettolo Marconi U. and Melchionna S., Langmuir, 28 (2012) 13727.

[21] Marconi U. M. B., Melchionna S. and PagonabarRAgA I., The Journal of Chemical Physics, 138 (2013) 244107.

[22] Pedone D., Firnkes M. and Rant U., Anal. Chem., 81 (2009) 9689.

[23] Chinappi M., Melchionna S., Casciola C. and Succi S., The Journal of Chemical Physics, 129 (2008) 124717.

[24] Bocquet L. and Charlaix E., Chemical Society Reviews, 39 (2010) 1073.

[25] Melchionna S. and Marconi U. M. B., EPL (Europhysics Letters), 95 (2011) 44002.

[26] Bernaschi M., Melchionna S., Succi S., Fyta M., Kaxiras E. and Sircar J. K., Computer Physics Communications, 180 (2009) 1495.

[27] Chinappi M. and Casciola C., Physics of Fluids, 22 (2010) 042003.

[28] Attard P., arXiv preprint arXiv:1302.6630, (2013) .

[29] Senapati S. and Chandra A., The Journal of Physical Chemistry B, 105 (2001) 5106. 